FORMATION Formation emploi

Revue française de sciences sociales

112 | octobre-décembre 2010

Pêle-mêle

\title{
Présentation de l'ouvrage : « Sociologie de la gestion. Les faiseurs de performance »
}

\section{Sophie Divay}

\section{(2) OpenEdition}

12 Journals

Édition électronique

URL : http://journals.openedition.org/formationemploi/3206

DOI : 10.4000/formationemploi.3206

ISSN : 2107-0946

Éditeur

La Documentation française

Édition imprimée

Date de publication : 30 décembre 2010

Pagination : 69-73

ISSN : 0759-6340

\section{Référence électronique}

Sophie Divay, «Présentation de l'ouvrage : «Sociologie de la gestion. Les faiseurs de performance » », Formation emploi [En ligne], 112 | octobre-décembre 2010, mis en ligne le 27 janvier 2011, consulté le 30 octobre 2020. URL : http://journals.openedition.org/formationemploi/3206 ; DOI : https://doi.org/ 10.4000/formationemploi.3206

(c) Tous droits réservés 


\title{
de lecture
}

\section{Présentation de l'ouvrage : "Sociologie de la gestion. Les faiseurs de performance»}

\author{
Sophie Divay*
}

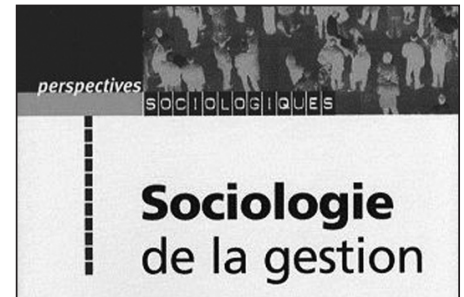

Les faiseurs de performance

VALÉRIE BousSARD

Dans cet ouvrage, Valérie Boussard répond à une question que tout le monde peut se poser : pourquoi la gestion se diffuse-t-elle partout? Après s'être répandue dans les grandes entreprises, elle se dissémine également au sein des services publics, des associations, de la sphère syndicale, voire même dans l'espace privé. Comme le note l'auteure, ce phénomène devient hégémonique et s'apparente désormais à un «fait social total».

Son émergence se produit à la fin du $\mathrm{XIX}^{\mathrm{e}}$ siècle, d'abord dans le monde des affaires et de la finance, puis dans celui de l'organisation du travail. La rationalité qu'elle introduit vise l'augmentation du profit, de la rentabilité et de la productivité. Une rupture s'opère avec les formes traditionnelles de « conduite des organisations »; le contrôle social moderne passe moins par un travail de surveillance que par une maîtrise des actions désormais finement mesurées. S'inspirant de l'ethos de l'entrepreneur capitaliste, cette maîtrise des actions a pour objectif la recherche du gain et du profit, et pour moyens le calcul, la prévoyance, l'abstraction. La devise de Saint-Simon, «le gouvernement des hommes et l'administration des choses ", trouve ainsi les fondements de son application concrète. Dans cette perspective, les rapports sociaux sont pacifiés puisque les décisions sont prises au nom de lois et mesures rationnelles, objectives et impersonnelles. Selon les thèses d'Adam Smith, reprises par l'économie politique classique, intérêt général et intérêt particulier se rejoignent, et le monde social est ainsi unifié.

Sophie Divay est sociologue, chargée d'études au Centre associé au Céreq de Paris rattaché au Centre d'Économie de la Sorbonne, université Paris 1. Elle a récemment publié deux articles portant sur certaines des incidences des politiques publiques de la santé et de l'emploi : "Les précaires du care ou les évolutions de la gestion de l'"absentéisme" dans un hôpital local ", Sociétés Contemporaines, n77, 2010 ; "Nouveaux opérateurs privés du service public de l'emploi: les pratiques des conseillers sont-elles novatrices? », Travail et Emploi, n 1 19, juillet-septembre, 2009. 
À l'issue de cette démarche rétrospective, l'auteure est en mesure de mieux définir ce qu'est la gestion : elle comprend un ensemble de dispositifs mobilisés pour conduire le fonctionnement des organisations. Les « modes de gouvernance », plus ou moins homogènes, sont régis par un même Logos gestionnaire constitué de trois principes fondamentaux : la maîtrise, la performance et la rationalité. Tel est l'étendard de la gestion. Dès lors, on pourrait croire le problème résolu : la généralisation et la légitimité de la gestion ne peuvent être dues qu'à son efficacité. Cette explication ne résiste cependant pas à l'épreuve du réel.

Le principe de la maîtrise et du contrôle s'avère tout d'abord inapplicable. Les travaux menés par l'auteure sur les indicateurs de gestion démontrent ainsi leurs limites et leur faible plus-value par rapport aux outils traditionnels qu'ils ont détrônés : le contrôle n'est pas meilleur qu'auparavant. La rationalité laisse également à désirer; de nombreuses enquêtes de terrain mettent au jour la fragilité des décisions prises par les responsables qui s'appuient davantage sur des « abrégés » que sur un examen approfondi de données détaillées.

La performance, enfin, demeure très difficile à objectiver. Les critères utilisés n'ont pas une valeur « en soi » ou absolue, mais relative aux objectifs et aux représentations de l'activité imposés à l'entreprise. Une dimension de la performance économique comme celle, a priori simple, de la rentabilité, recouvre des critères variables et souvent contradictoires : un dispositif de gestion faisant gagner des parts de marché peut entraîner une perte de chiffre d'affaires; un autre dispositif permettant une meilleure productivité peut provoquer une baisse de la qualité des marchandises ou services produits.

Pourquoi la gestion conserve-t-elle sa légitimité malgré son inefficacité ? En fait, elle doit sa pérennité à la croyance qu'elle suscite. Seule la performativité du Logos gestionnaire permet une telle adhésion non démontrable, mais justifiée à l'aide d'arguments qui affirment la neutralité, la justesse et la vertu de la gestion. Elle est neutre parce qu'elle obéit à des lois impersonnelles et indiscutables (soumission aux exigences du marché pour le bien de l'entreprise); juste, parce que scientifiquement fondée ; vertueuse, parce qu'elle incite à l'autorégulation des comporte- ments plutôt qu'à l'exercice d'une surveillance infantilisante des salariés.

«Mais si la gestion ne fait pas ce qu'elle annonce, que fait-elle? ». Se réduit-elle à une forme idéelle? À un discours performatif? N'a-t-elle que des effets symboliques? Non, elle a également des effets bien tangibles, mais dissimulés. Valérie Boussard rapporte de nombreuses observations de terrain qui témoignent d'une disciplinarisation du travail, c'est-à-dire d'un contrôle des subjectivités. Les salariés sont soumis à une prescription d'autonomie qui les contraint entre autres à se sortir d'eux-mêmes de situations de travail paradoxales.

Reste toutefois à savoir qui tire les ficelles de tels mécanismes d'assujetissement. Selon la thèse défendue par Luc Boltanski et Eve Chiapello (1999), les dispositifs de gestion seraient les instruments idéologiques du capitalisme, d'autant plus efficaces qu'ils favoriseraient l'engagement des travailleurs.

En examinant de plus près «comment se fait la gestion », des sociologues des organisations nous apprennent que les techniques de management ne s'appliquent pas sur des acteurs passifs. Ceux-ci disposent d'un pouvoir de résistance et mettent en œuvre des stratégies défensives, plus ou moins efficaces. La rationalisation des pratiques est le fruit d'ajustements et de négociations locales.

Selon Valérie Boussard, ces deux interprétations d'une même réalité mènent à une impasse. Dans un cas, les dispositifs de gestion sont vus comme imposés d'en haut ; dans l'autre, ils résultent d'arrangements construits tout en bas de l'organisation. Rien n'est alors éclairci : on ne sait pas qui pilote en haut; on ignore comment s'articulent, en bas, le Logos gestionnaire et les micro-pratiques locales disparates.

Les écrits de Michel Foucault (1969) sur l'univers médical offrent à l'auteure une troisième voie d'investigation. Pour comprendre les ressorts du discours de la médecine clinique à la fin du XVIII ${ }^{\mathrm{e}}$ siècle, le philosophe interroge les positions et fonctions institutionnelles des médecins, c'est-à-dire le niveau intermédiaire entre le Logos et la Praxis. De la même manière, l'auteure choisit de s'intéresser aux gestionnaires, placés entre le niveau macro-social abstrait et le niveau micro-social concret. Ces professionnels 
ont une position centrale et incarnent tout à la fois les « légitimateurs » et les « diffuseurs » de la gestion.

Cette orientation constitue un véritable tournant dans cet ouvrage, tournant que le sous-titre (« Les faiseurs de performance ») annonce allusivement. On passe ainsi d'une sociologie de la gestion à une sociologie des gestionnaires. Qui sont-ils donc ? Ils se répartissent dans trois catégories. La première, celle des managers, comprend des ingénieurs, des comptables et un personnel d'encadrement; la deuxième est composée d'enseignants-chercheurs; la troisième de consultants. Les gestionnaires sont issus d'écoles d'ingénieurs, d'écoles de commerce, de l'université (notamment Paris Dauphine), de formations conçues sur le modèle de la Business School américaine, ou encore titulaires d'un $\mathrm{PhD}$ obtenu au États-Unis (Harvard ou Stanford) pour les membres de l'élite des enseignants de gestion. Ils ont donc suivi des formations différentes et occupent également des emplois divers, dans le privé et le public ou en libéral. Leur milieu n'est toutefois pas composé de segments cloisonnés. Les individus sont mobiles et se déplacent entre l'entreprise, l'enseignement et le conseil, mobilité qui facilite la circulation et la constitution des savoirs dans ce domaine.

La diversité du milieu rend toutefois complexe son identification. Il ne saurait être considéré comme un groupe professionnel homogène, et l'auteure préfère de ce fait se pencher sur les processus de professionnalisation qui l'animent. Si ce processus s'est traduit par la création d'associations professionnelles et de revues de gestion et de management, il n'a pas encore débouché sur la conquête d'un monopole légal d'exercice. Le marché du travail de la gestion demeure imparfaitement fermé, mais pour autant pas totalement non régulé. Il existe une hiérarchie des diplômes qui sont classés selon leur degré de prestige. La valeur socioprofessionnelle d'un gestionnaire ne dépend cependant pas que de son diplôme, mais aussi de la réputation des employeurs qui l'ont embauché.

Si ce milieu n'est pas homogène, il n'est pas non plus atone. Il est le théâtre de luttes ou de "compétitions interprofessionnelles » (pour reprendre une expression d'Andrew Abbott, 1988) qui se jouent autour de la reconnaissance du savoir gestionnaire. Elle sera octroyée à ceux qui auront fait la démonstration qu'ils détiennent la meilleure solution $\mathrm{au}(\mathrm{x})$ problème(s) des clients. Ces clients ne forment pas davantage un groupe homogène. Les consultants et enseignantschercheurs ciblent les managers auxquels ils vendent leurs dispositifs de gestion ; les managers en entreprise, quant à eux, doivent persuader les membres de leur organisation de l'efficacité des dispositifs qu'ils veulent introduire dans l'entreprise.

Les consultants et enseignants-chercheurs proposent des produits - démarches, outils, dispositifs, procédures, etc. - qui ont des cycles de vie ou, pour le dire autrement, suivent des modes managériales. Celles-ci évoluent au gré non seulement des demandes des clients, mais aussi du travail de mise en forme symbolique et matérielle des produits, c'est-à-dire d'opérations de packaging. Les " gestionnaires-prescripteurs » (consultants et enseignants-chercheurs chargés d'une mission de conseil), qui réalisent ces opérations, sont en concurrence et mènent de ce fait des luttes de territoire. Si la relation avec l'institution est d'ordre marchand pour les prescripteurs, elle est d'une autre nature pour les managers. Ces derniers sont en effet confrontés à des attentes de rôle très exigeantes, puisqu'ils sont censés incarner les principes du Logos gestionnaire. "Jouer le manager » signifie notamment adopter les techniques de management les plus en vogue, non pas tant pour atteindre l'efficacité que pour mettre en scène un souci d'efficacité et ainsi gagner en légitimité. Il s'agit de jouer sur les apparences et de mettre en œuvre un « simulacre d'utilisation des dispositifs ». En cas d'échec, le manager peut ainsi objecter qu'il a mis en pratique les techniques et procédures en vigueur, qu'il s'est conformé aux attentes normatives de son milieu.

Ces deux types de dynamiques professionnelles gestionnaires, celle des enseignants-chercheurs et consultants, d'une part, et celle des managers, d'autre part, bien que différentes, contribuent l'une comme l'autre à la diffusion de la gestion. La première de ces dynamiques s'inscrit au sein d'un marché de prescripteurs dont l'objectif est d'encourager la consommation de dispositifs de gestion en perpétuel renouvellement. La seconde est propre au groupe professionnel des managers : ceux-ci sont d'autant plus contraints de tenir leur rôle qu'ils sont, en tant que décideurs, confrontés à l'incertitude qui les pousse au conformisme. Par le biais d'un « isomorphisme mimétique », 
ils limitent leur prise de risque, et donc adoptent les mêmes dispositifs que ceux utilisés par leurs homologues. L'auteure souligne qu'ils vérifient ainsi une règle déjà observée par Keynes selon laquelle « $L a$ sagesse universelle enseigne qu'il vaut mieux pour sa réputation échouer avec les conventions que réussir contre elles » (p. 217).

La diffusion des dispositifs de gestion emprunte encore d'autres voies, et notamment celles que les gestionnaires construisent à travers leur circulation entre différentes institutions. Des échanges ont lieu entre universités et cabinets-conseils, entre cabinetsconseils et entreprises, et enfin entre universités et entreprises. Un brassage et une harmonisation des connaissances, normes et conventions se produisent à l'occasion de rencontres dans le cadre de séminaires, formations, manifestations d'associations professionnelles, etc. Les acteurs codéfinissent ainsi les problèmes à traiter et les solutions que la gestion peut apporter. Ils nouent des alliances et stabilisent un socle commun de connaissances et de conventions.

L'hypothèse selon laquelle la diffusion de la gestion serait le fait du capitalisme est donc définitivement invalidée. Le rôle central des gestionnaires a été mis au jour. L'efficacité de leur action réside notamment dans la portée de leur rhétorique professionnelle qui contribue à la délimitation et à la défense des frontières de leur territoire. Toutefois, il ne faudrait pas en conclure que la gestion n'a rien à voir avec le capitalisme. Elle participe au contraire pleinement à l'ancrage et à la consolidation des catégories de l'économie capitaliste.

Valérie Boussard offre aux lecteurs un précieux outil de compréhension de la gestion, de ses dispositifs et plus largement des nouvelles technologies de pouvoir qui habitent désormais toutes les sphères du travail.
Son ouvrage ne sera certainement pas sans produire des «résonances biographiques» (Strauss, 1992) chez les travailleurs aux prises avec les effets du management dont le Logos gestionnaire occulte les logiques sous-jacentes. Un autre apport réside dans la contribution que les analyses de l'auteure apportent à la sociologie des groupes professionnels en s'intéressant à un espace professionnel difficile à cerner. Elle fournit un modèle analytique d'un objet multiple et segmenté, situé à des années lumière de la conception mécaniste du fonctionnalisme. Elle prend à bras le corps toute la complexité d'une activité exercée par des professionnels occupant des statuts et des emplois très différents. Elle réussit à objectiver leurs liens d'interdépendance, tout en prenant en compte la dimension historique, appliquant ainsi une « approche configurationnelle », telle que prônée par Norbert Elias et John L. Scotson (2001).

On ne peut que souhaiter que Valérie Boussard continue à creuser ce sillon, en lui suggérant par exemple d'explorer davantage deux pistes. Tout d'abord, celle des effets directs et indirects que l'application des dispositifs de gestion peut avoir sur les travailleurs. Même si l'efficacité de ces dispositifs n'est qu'un leurre, comme le démontre l'auteure, ils ne sont pas sans peser fortement sur les pratiques des salariés, leur collectif et leur rapport au travail. Ils sont coûteux en énergie, en temps et perturbent leur « définition du travail bien fait », aspect abordé un peu trop rapidement dans cet ouvrage. L'autre piste est celle des connexions existantes entre les gestionnaires, ou certaines catégories de gestionnaires, et la sphère politique. Si le capitalisme est amplement pris en compte dans l'ouvrage, il l'est peut-être trop sous son angle économique, au détriment des enjeux qui se font jour dans les arènes du pouvoir politique. 


\section{Référence de l'ouvrage}

Boussard V. (2008), Sociologie de la gestion. Les faiseurs de performance, Paris, Éditions Belin.

\section{Bibliographie}

Abbott A. (1988), The system of Professions. An Essay on the Division of Expert labour, ChicagoLondres, University of Chicago Press.

Boltanski L. et Chiapello E. (1999), Le nouvel esprit du capitalisme, Paris, Gallimard.

Elias N., Scotson J.L. (2001), Les logiques de l'exclusion, Paris, Pocket.
Foucault M. (1969), L'archéologie du savoir, Paris, Gallimard.

Strauss A.L. (1992), Masques et miroirs. Une introduction à l'interactionnisme, Paris, Éditions Métaillé. 